\title{
Variación estacional y características fisicoquímicas e hidrológicas que influyen en los macroinvertebrados acuáticos, en un río tropical
}

\author{
Francisco Quesada-Alvarado*1, Gerardo Umaña Villalobos ${ }^{2}$, Monika Springer ${ }^{3}$ \\ \& Jorge Picado Barboza ${ }^{4}$ \\ 1. Programa Regional de Posgrado en Biología, Sistema de Estudio de Posgrado, Escuela de Biología, Universidad de \\ Costa Rica (UCR), San José, Costa Rica \& Instituto Regional de Estudios en Sustancias Tóxicas (IRET), Universidad \\ Nacional (UNA), Heredia, Costa Rica francisco.quesada.alvarado@una.ac.cr \\ 2. Centro de Investigación en Ciencias del Mar y Limnología (CIMAR), Universidad de Costa Rica (UCR), San José, \\ Costa Rica. gerardo.umana@ucr.ac.cr \\ 3. Escuela de Biología \& CIMAR, Universidad de Costa Rica, San José, Costa Rica. Monika.springer@ucr.ac.cr \\ 4. Instituto Costarricense de Electricidad (ICE), San José, Costa Rica. jorgepicadobioconsult@yahoo.com
}

$$
\text { Recibido 30-X-2019. Corregido 07-VII-2020. Aceptado 31-VIII-2020. }
$$

\begin{abstract}
Seasonal variation, physicochemical and hydrological characteristics that influence aquatic macroinvertebrates, in a tropical river. Introduction: The distribution of aquatic macroinvertebrates in rivers may be determined by the type of substrate, the shape of the river channel, the hydrological regime, and interactions with biotic and abiotic factors. Variations in the distribution of aquatic macroinvertebrates due to transitions and variations in physicochemical and hydrological characteristics are unknown in most rivers. Objectives: 1) to study the change in the aquatic macroinvertebrates assemblages in two sites in Naranjo River, during the four hydrological seasons; 2) to determine the relationship between the physicochemical variables, substrate and flow with the assemblages. Methodology: The study was conducted in the Naranjo River, Costa Rica, at two sampling sites during one year, evaluating the four seasons of the year. In each station we measure, values of water velocity and depth, flow rate, dissolved oxygen, $\mathrm{pH}$, conductivity, sulfates, ammonium, nitrate, silicates, phosphates, and substrate were obtained, and a sample of aquatic macroinvertebrates was obtained for each type of microhabitat determined by water velocity. Two-way PERMANOVA was made to determine if there is a difference among aquatic macroinvertebrate abundance, per period and between sites. Canonical correspondence analysis was used to observe the relationship between aquatic macroinvertebrates and environmental factors. Finally, a Spearman's rank correlation coefficient was performed to determine the positive or negative relationship of variables with macroinvertebrates. Results: There was a greater abundance during the dry season, while during the transition from dry to the rainy season, the lowest abundance of organisms was recorded. The CCA indicates that the first axis presented a positive relationship with the silt and sand substrate and a negative relationship for the current velocity and rock substrate variables. The second axis showed a positive relationship with flow and nitrates and negative for the pebble substrate. Most organisms showed a negative response to increased flow and nitrates. Discussion: As flow increases, current velocity increases, and not all organisms recorded in the Naranjo River have the characteristics to survive these conditions. In addition, the depth also increases along with the drag force, causing movement of smaller substrates and removal of organisms. Increased nitrates can lead to a decrease in more sensitive organisms, such as Plecoptera and Ephemeroptera. In spite of the physicochemical and flow variations registered in the Naranjo River, these were not strong enough to vary the assemblages of aquatic macroinvertebrates, so the basin does not have stressful factors that eliminate organisms during one or several months of the year.
\end{abstract}

Keywords: flow; current velocity; substrate; aquatic macroinvertebrate assemblages; aquatic insects.

Quesada-Alvarado, F., Umaña Villalobos, G., Springer, M., \& Picado Barboza, J. (2020). Variación estacional y características fisicoquímicas e hidrológicas que influyen en los macroinvertebrados acuáticos, en un río tropical. Revista de Biología Tropical, 68(Supl. 2), S54-S67. 
Los macroinvertebrados acuáticos (MIA) son uno de los grupos más representativos en los ríos y su estructura y distribución puede estar mediada por procesos bióticos como la interacción por depredación, parasitismo o competencia (Molina, Gibon, Pinto, \& Rosales, 2008; Ferru \& Fierro, 2015; Roldán-Pérez, 2016). Además, existen procesos y factores abióticos que determinan la abundancia y distribución de los invertebrados en los ríos (Fonnesu, Sabetta \& Basset, 2005; Harinkhede \& Manekar, 2016). En este aspecto, las propiedades físicas y químicas del agua pueden influir en la presencia de determinados grupos, dependiendo de la sensibilidad del organismo ante una o varias variables (Kail, Arle, \& Jähnig, 2012). Por ejemplo, la concentración de oxígeno disuelto y la demanda biológica de oxígeno, puede condicionar la presencia o ausencia de macroinvertebrados acuáticos (Kubendran \& Ramesh, 2016). La variación en el caudal es otro componente que cambia la distribución y abundancia de los macroinvertebrados acuáticos. Así, ante disminuciones del caudal se da un aumento de organismos que toleran bajas concentraciones de oxígeno y altas temperaturas (Extence et al. 1999). Con el aumento del caudal y el consecuente aumento en las velocidades y del oxígeno disuelto, se favorecen a aquellas especies que están adaptadas a altas velocidades y mayor fuerza de arrastre (Horrigan \& Baird, 2008).

Las variaciones en las características fisicoquímicas e hidrológicas de un río son más marcadas cuando hay presencia de una actividad humana, por ejemplo, la agricultura, el urbanismo, la minería y las represas, las cuales ponen en riesgo a la integridad ecológica (Vörösmarty et al., 2010). No obstante, los cambios ambientales también proceden de manera natural producto de la escorrentía y las variaciones en el caudal (Allan \& Castillo, 2007). De esta forma, dependiendo de la región, las variaciones se pueden dar de forma diaria y marcada en los ríos tropicales y van a depender ya sea por el deshielo de glaciares o por precipitaciones intermitentes, provocando cambios constantes en la estructura de los ensamblajes acuáticos (Syvitski, Cohen, Kettner \& Brakenridge, 2014; Cauvy-Fraunié et al., 2016).

La relación entre los macroinvertebrados acuáticos con variables fisicoquímicas e hidrogeomorfológicas, ha permitido utilizarlos como indicadores de la condición de los sistemas acuáticos (Appalasamy, Arumugam, Sukri, \& Rak, 2018; Mengzhen, et al. 2018; Sefick, Kosnicki, Paller, \& Feminella, 2018). Por ejemplo, en Costa Rica, los estudios relacionados con estos organismos y su rol como bioindicadores han aumentado recientemente en programas de monitoreo de la calidad biológica del agua (Springer, 2019). Sin embargo, se desconoce en gran parte de los ríos, las variaciones en la distribución y abundancia de los macroinvertebrados acuáticos producto de las transiciones anuales y consecuentes variaciones en características fisicoquímicas e hidrológicas, que no procedan de actividades antropogénicas.

Los objetivos de este estudio fueron: 1) estudiar el cambio del ensamblaje de macroinvertebrados acuáticos en dos sitios dentro del cauce del Río Naranjo, durante los cuatro periodos hidrológicos del año; y 2) determinar la relación entre las variables fisicoquímicas, sustrato y el caudal con el ensamblaje de MIA. Nuestra hipótesis fue que, los cambios en las variables fisicoquímicas e hidrológicas al pasar de una estación climática a otra alteran la composición del ensamblaje de MIA. Con la predicción de una disminución en la abundancia al pasar de un periodo a otro y al variar los parámetros fisicoquímicos e hidrológicos. Contribuyendo a entender la variación de los MIA en el Río Naranjo, como línea base para trabajos de manejo de cuencas y posibles afectaciones por aprovechamiento del recurso hídrico o cambio del uso del suelo aguas arriba o en las cercanías.

\section{MATERIALES Y MÉTODOS}

Para este trabajo se seleccionó la cuenca del Río Naranjo, ubicada en la vertiente del Pacífico Central de Costa Rica. Esta cuenca posee una red de drenaje de $323.39 \mathrm{~km}^{2}$, lo que corresponde a un $0.63 \%$ de la superficie 
nacional (Proyecto de Ley 20-098, 2018). La cuenca cuenta con poca intervención antrópica, la principal actividad a su alrededor es agrícola y no existen represas hidroeléctricas (según El Sistema Nacional de Información para la Gestión Integrada del Recurso Hídrico, del Ministerio de Ambiente y Energía de Costa Rica), lo cual le confiere el carácter de una cuenca de referencia.

Se realizaron un total de diez muestreos, hasta completar un ciclo anual donde se evaluaron los cuatro periodos hidrológicos del año (seco, lluvioso, transiciones de seco a lluvioso y de lluvioso a seco (no hubo muestreo en el mes de julio 2017 debido a un robo en el equipo y en el mes de octubre 2017 debido a la tormenta Nate). Se seleccionaron dos sitios de muestreo ambos en un tramo no mayor a los 100 metros, el primero denominado N1 (9.5120778, -84.0334194) ubicado en la cuenca media-baja a 100 m.s.n.m. y un segundo sitio, denominado N2 (9.4629111, -84.0679361), en la cuenca media a 620 m.s.n.m; ambos dentro del cauce principal del Río Naranjo (Fig. 1). El primer sitio, se caracteriza por la presencia de sistemas tipo rápidos moderados y pozas; el sustrato está dominado por cantos rodados y grava, hay contacto del cuerpo de agua con la vegetación de ribera, la cual está conformada por árboles dispersos y gramíneas. En el segundo sitio, N2, tiene mayor pendiente, predominando los sistemas tipo rápidos turbulentos,

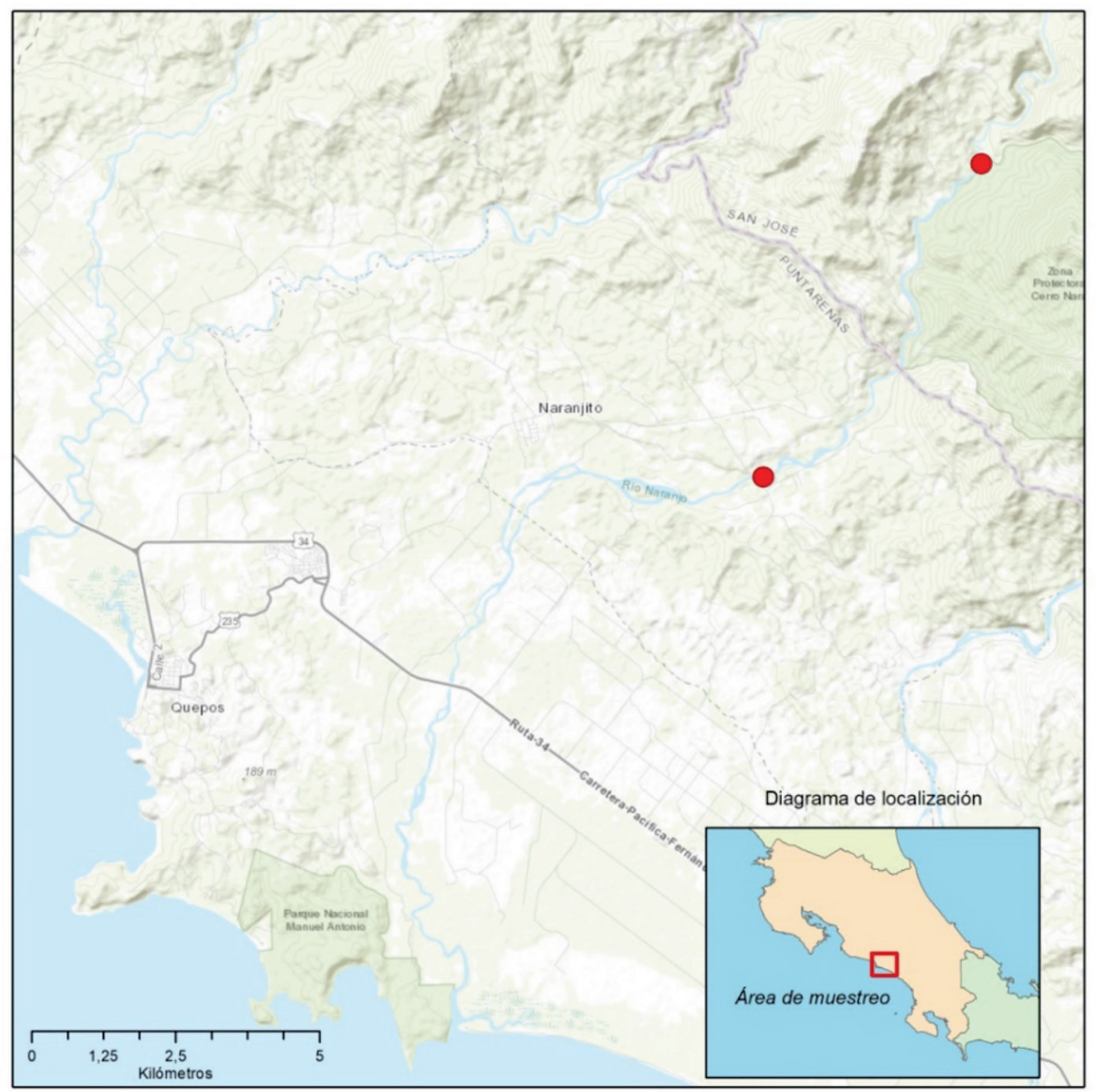

Fig. 1. Zona de estudio, en el cauce principal del río Naranjo, Quepos, Costa Rica, 2017-2018. Fig. 1. Study zone, in the main channel of the Naranjo River, Quepos, Costa Rica, 2017-2018. 
con dominancia de sustratos tipo canto rodado y roca, ambas márgenes se encuentran cubiertas con árboles y arbustos.

En cada sitio de muestreo y por mes se recolectaron un total de diez muestras (diez réplicas por sitio), las cuales se muestrearon de manera aleatoria, de esta forma, en periodo seco y en transición de seco a lluvioso, se iniciaba en el margen izquierdo y se finalizaba en el margen derecho, mientras que en el periodo lluvioso y de transición lluvioso a seco, se iniciaba en el margen izquierdo y se iba ingresando de manera transversal al río, hasta donde se pudiera muestrear debido al aumento de caudal y corriente. De no completar las diez muestras de manera transversal, se procedía a subir o bajar como mínimo 10 metros de manera longitudinal al río y nuevamente, se ingresaba de manera transversal.

En cada microhábitat (muestra) se sumergió un velocímetro marca Global Water, se determinaba la velocidad (al 60\% del fondo) y profundidad del agua. Seguidamente, se introdujo una red D de 500 micras de abertura de malla, $30 \mathrm{~cm}$ de ancho y $27 \mathrm{~cm}$ de alto, se removía el sustrato frente a la red por 35 segundos, para que los organismos fueran arrastrados y atrapados en la red. El material atrapado se depositó en una bolsa plástica debidamente rotulada con el número de microhábitat y así sucesivamente para cada réplica. El material se preservó con etanol al $85 \%$, para su posterior separación e identificación taxonómica en el laboratorio. De igual forma en cada microhábitat evaluado se obtuvieron los valores de $\mathrm{pH}$, temperatura $\left({ }^{\circ} \mathrm{C}\right)$, oxígeno disuelto $(\mathrm{mg} / \mathrm{l})$ y el porcentaje de cada tipo de sustrato, catalogado como roca, canto rodado, grava, arena, limo (siguiendo la clasificación de American Geophysical Union, 2016) y los paquetes de hojas los cuales fueron clasificados como un tipo de sustrato.

En cada sitio de muestreo (una vez finalizado el muestreo de macroinvertebrados) se recolectó una muestra de agua en una botella color ámbar de $500 \mathrm{ml}$, para análisis de nitritos, nitratos, amonio, fosfatos y silicatos, que fue trasladada con hielo al laboratorio químico del
Centro de Investigación en Ciencias del Mar y Limnología (CIMAR) de la Universidad de Costa Rica, mientras que, la conductividad eléctrica se midió en el campo. Los valores de nitritos, nitratos, fosfatos, silicatos y amonio se obtuvieron a través de un autoanalizador de flujo continuo marca Lachat, Modelo QuickChem 8500. El caudal del río en el momento preciso del muestreo fue obtenido a través de estaciones hidrológicas automáticas y los datos fueron brindados por el Instituto Costarricense de Electricidad (ICE).

Los macroinvertebrados acuáticos recolectados fueron identificados al menor nivel taxonómico posible, en su mayoría a género, con excepción del grupo Hidrachnidia, por medio de las claves taxonómicas: Contreras \& Harris (1998), Roldán (1998), Manzo \& Archangelsky (2008), Flowers \& De la Rosa (2010), Ramírez (2010) y Springer (2010). Los organismos fueron depositados en la colección de Entomología Acuática del Museo de Zoología de la Universidad de Costa Rica (MZUCR).

Análisis estadístico: Para determinar diferencia de la abundancia de macroinvertebrados acuáticos por periodo hidrológico del año y entre los dos sitios, se realizó un PERMANOVA de dos vías, con distancia euclidiana. Previo al análisis se evaluó la normalidad de la abundancia y las variables ambientales. Dado que ninguna presentó normalidad se procedió a realizar pruebas no paramétricas. Con el paquete Vegan (Oksanen, 2019), se realizó un análisis de correspondencia canónica (ACC), para determinar asociaciones entre la estructura de la comunidad (géneros de macroinvertebrados acuáticos) y las variables ambientales: velocidad, profundidad, temperatura, nitritos, nitratos, fosfatos, silicatos, amonio, conductividad, tipo de sustrato y caudal $\left(\mathrm{m}^{3} / \mathrm{s}\right)$. En el análisis ACC se utilizaron variables ambientales no correlacionadas, lo cual se evaluó con el paquete ggcorrplot (Kassambara, 2019). Antes de realizar el análisis se transformó a escala logarítmica las variables, caudal, silicatos y conductividad con el fin de estandarizarlas con el resto de las variables. Seguidamente 
con las variables de mayor peso se aplicó una prueba de Spearman para determinar su grado de relación (positivo o negativo) y los valores de la misma fueron graficados en un mapa de calor, a través del paquete ggplot2 (Wickham, 2016). Todas las pruebas se realizaron a través del software R (R Core Team, 2019).

\section{RESULTADOS}

Los valores más altos de fosfatos, silicatos, amonio, nitratos y de caudal se registraron durante los meses con mayores precipitaciones (Tabla 1). Únicamente los nitritos registraron su mayor concentración en el segundo muestreo correspondiente al periodo seco. La velocidad mínima y máxima registrada respectivamente en el sitio $\mathrm{N} 1$ fue de $0 \mathrm{~m} / \mathrm{s}$ y 2.0 $\mathrm{m} / \mathrm{s}$, y en el N2 fue de 0 y $2.1 \mathrm{~m} / \mathrm{s}$. El valor de oxígeno disuelto varió entre $6.8 \mathrm{mg} / \mathrm{l}$ y $8.8 \mathrm{mg} / \mathrm{l}$ en ambos sitios (Tabla 1).

Entre los dos sitios muestreados, se evaluaron un total de 230 muestras y se identificaron 7742 organismos, en 75 géneros, 38 familias y 11 órdenes de macroinvertebrados acuáticos; siendo el género Camelobaetidius sp. (Ephemeroptera: Baetidae) el más abundante en ambos sitios. De igual forma, la familia Baetidae (12 géneros) y el orden Ephemeroptera, (20 géneros) presentaron la mayor riqueza taxonómica (Fig. 2 C y Fig. 2 D). En cuanto a la abundancia por orden, se observó que los más abundantes en ambos sitios fueron

TABLA 1

Valores de las variables fisicoquímicas, caudal y temperatura de los sitios de muestreo en la cuenca del Río Naranjo, 2017-2018. $\mathrm{S}=$ sitio, $\mathrm{Q}=$ Caudal, $\mathrm{C}=\mathrm{Seco}, \mathrm{T}=$ Transición, $\mathrm{L}=$ Lluvioso, Inc=Incertidumbre, $\mathrm{nq}=$ no cuantificable, $\mathrm{nd}=$ no detectable

TABLE 1

Values of the physicochemical variables, water flow and temperature of the sampling sites in the Naranjo River basin, 2017-2018 S = site, Q= Flow, C= Dry, T= Transition, L= Rainy, Inc= Uncertainty, nq= not quantifiable, nd= not detectable

\begin{tabular}{|c|c|c|c|c|c|c|c|c|c|c|c|c|c|}
\hline Mes & S & $\begin{array}{l}\text { Fosfatos } \\
(\mu \mathrm{mol} / \mathrm{L})\end{array}$ & $\operatorname{Inc} \pm$ & $\begin{array}{c}\text { Silicatos } \\
(\mu \mathrm{mol} / \mathrm{L})\end{array}$ & Inc \pm & $\begin{array}{c}\text { Amonio } \\
(\mu \mathrm{mol} / \mathrm{L})\end{array}$ & $\operatorname{Inc} \pm$ & $\begin{array}{l}\text { Nitritos } \\
(\mu \mathrm{mol} / \mathrm{L})\end{array}$ & $\operatorname{Inc} \pm$ & $\begin{array}{l}\text { Nitratos } \\
(\mu \mathrm{mol} / \mathrm{L})\end{array}$ & $\operatorname{Inc} \pm$ & $\mathrm{Q}\left(\mathrm{m}^{3} / \mathrm{s}\right)$ & $\mathrm{C}^{0}$ \\
\hline Enero 2017 & N1 & 0.47 & 0.12 & 121.5 & 1.27 & 5.22 & 0.22 & nd & 0.4 & 1.5 & 0.37 & 8.94 & 25.37 \\
\hline (C) & N2 & 0.18 & 0.12 & 117.75 & 1.24 & 3.92 & 0.22 & nd & 0.4 & 2.76 & 0.36 & 8.16 & 21.5 \\
\hline Febrero & N1 & 0.54 & 0.12 & 120.75 & 1.27 & 2.72 & 0.22 & nd & 0.4 & 1.74 & 0.37 & 13.86 & 26.35 \\
\hline (C) & $\mathrm{N} 2$ & $\mathrm{nq}$ & 0.12 & 120.9 & 1.27 & 2.83 & 0.22 & 4.15 & 0.4 & nd & 0.36 & 13.3 & 21.09 \\
\hline Marzo & N1 & 0.22 & 0.12 & 120.03 & 1.27 & 8.24 & 0.22 & nd & 0.4 & 0.63 & 0.37 & 9.65 & 25.5 \\
\hline (C) & N2 & 0.25 & 0.12 & 120.39 & 1.27 & 3.6 & 0.22 & nd & 0.4 & 2.68 & 0.37 & 6.99 & 21.59 \\
\hline Abril & N1 & 0.28 & 0.12 & 112.78 & 1.27 & 3.53 & 0.22 & nd & 0.4 & 1.58 & 0.37 & 17.5 & 24.84 \\
\hline (C) & N2 & 0.29 & 0.12 & 118.39 & 1.27 & 3.67 & 0.22 & nd & 0.4 & 0.71 & 0.37 & 16.8 & 21.97 \\
\hline Mayo & N1 & 0.54 & 0.12 & 115.42 & 1.27 & 4.41 & 0.22 & nd & 0.4 & 3.39 & 0.37 & 27.9 & 23.9 \\
\hline$(\mathrm{T})$ & N2 & 0.48 & 0.12 & 118.73 & 1.27 & 5.11 & 0.22 & nd & 0.4 & 3.77 & 0.37 & 27.7 & 22.88 \\
\hline $\begin{array}{l}\text { Junio } \\
\text { (L) }\end{array}$ & N1 & 0.74 & 0.12 & 80.19 & 1.27 & 4.02 & 0.22 & 0.88 & 0.4 & 3.3 & 0.37 & 19.25 & 22.91 \\
\hline Agosto & N1 & 0.74 & 0.12 & 120.2 & 1.27 & 4.27 & 0.22 & 0.93 & 0.4 & 1.77 & 0.37 & 41.5 & 23.12 \\
\hline (L) & N2 & 0.7 & 0.12 & 86.81 & 1.27 & 3.85 & 0.22 & 0.64 & 0.4 & 3.97 & 0.37 & 38 & 21.3 \\
\hline Setiembre & $\mathrm{N} 1$ & 1.04 & 0.12 & 102.41 & 1.27 & 5.43 & 0.22 & 1.23 & 0.4 & 2.79 & 0.37 & 42.8 & 22.9 \\
\hline (L) & N2 & 1.04 & 0.12 & 116.72 & 1.27 & 5.85 & 0.22 & 1.42 & 0.4 & 3.73 & 0.37 & 41.5 & 21.7 \\
\hline Octubre & N1 & 1.05 & 0.12 & 104.86 & 1.27 & 5.29 & 0.22 & $\mathrm{nq}$ & 0.4 & 4.64 & 0.37 & 27.3 & 22.9 \\
\hline (L) & N2 & 0.66 & 0.12 & 94.51 & 1.27 & 3.81 & 0.22 & nd & 0.4 & 5.9 & 0.37 & 26.8 & 21.7 \\
\hline Noviembre & $\mathrm{N} 1$ & $\mathrm{nq}$ & 0.12 & 120.47 & 1.27 & 4.41 & 0.22 & nd & 0.4 & 4.1 & 0.37 & - & 19.2 \\
\hline (T) & $\mathrm{N} 2$ & 0.31 & 0.12 & 120.81 & 1.27 & 5.43 & 0.22 & nd & 0.4 & 3.26 & 0.37 & - & 19.67 \\
\hline $\begin{array}{l}\text { Enero } 2018 \\
\text { (S) }\end{array}$ & N1 & $\mathrm{nq}$ & 0.12 & 120.48 & 1.27 & 4.41 & 0.22 & nd & 0.4 & 4.1 & 0.35 & 9.5 & 21.7 \\
\hline
\end{tabular}



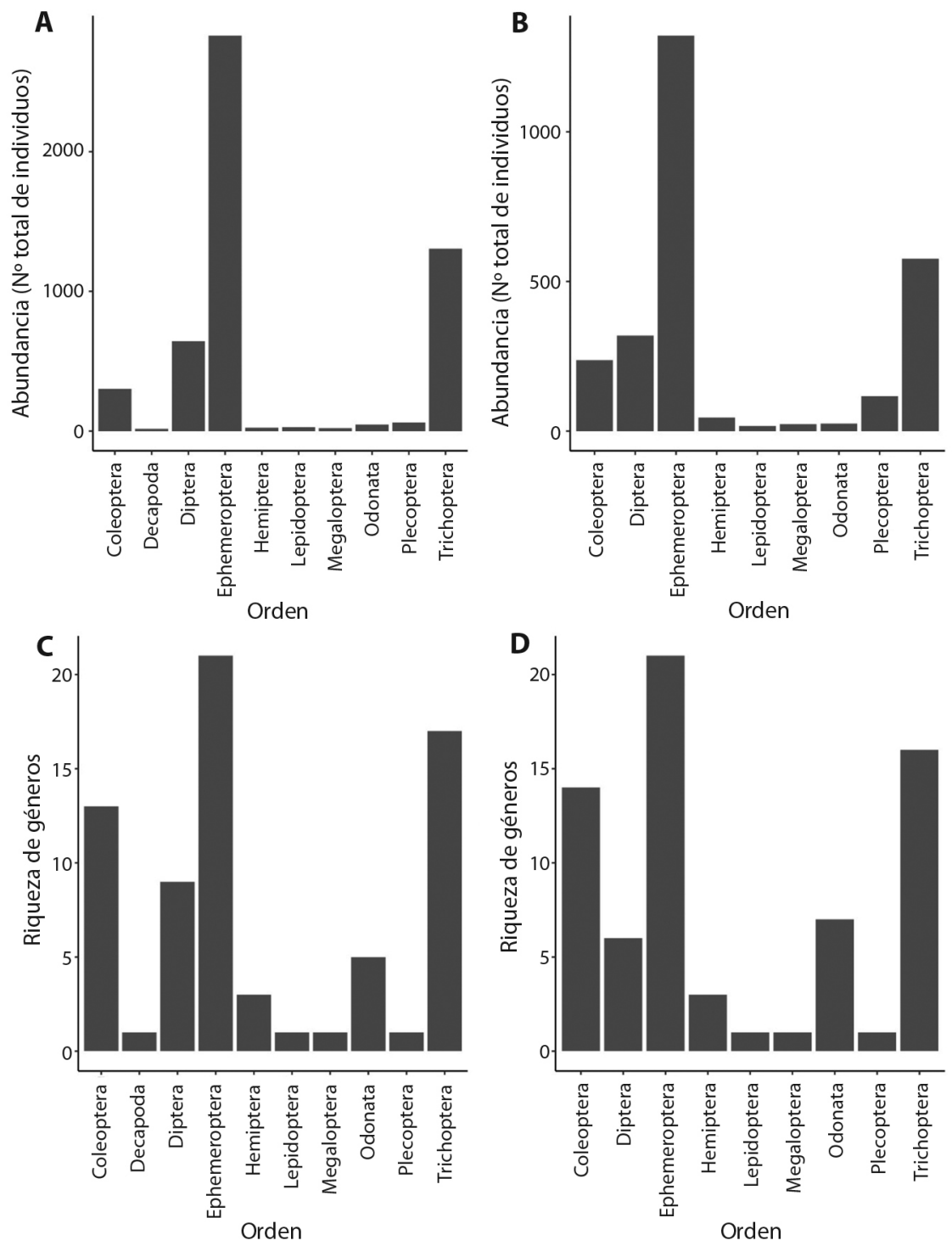

Fig. 2. Abundancia y riqueza de géneros por orden de macroinvertebrados acuáticos, A) Abundancia de macroinvertebrados acuáticos en el sitio N1; B) Abundancia de macroinvertebrados en el sitio N2; C) Riqueza de macroinvertebrados acuáticos en el sitio N1; C) Riqueza de macroinvertebrados acuáticos en el sitio N2, Río Naranjo, Costa Rica, 2017-2018.

Fig. 2. Abundance and richness of genera by order of aquatic macroinvertebrates, A) Aquatic macroinvertebrate abundance in the site N1; B) Aquatic macroinvertebrate abundance in the site N2; C) Aquatic macroinvertebrate richness in the site N1; D) Aquatic macroinvertebrate richness in the site N2, Naranjo River, Costa Rica, 2017-2018.

Ephemeroptera, Trichoptera y Diptera (Fig. 2 A y Fig. 2 B). Únicamente en el sitio N1 se registró al orden Decapoda, mientras que, el resto de los órdenes se encontraron en ambos sitios.

Para ambos sitios de muestreo, la abundancia de macroinvertebrados acuáticos varió según el periodo del año, de esta forma, se observó una mayor abundancia durante el periodo seco, mientras que, durante la transición de seco a lluvioso, se registró la menor abundancia de organismos (PERMANOVA, $\mathrm{F}=174.989 ; \mathrm{p}=0.001 ;$ Fig. 1). Además, no hubo 

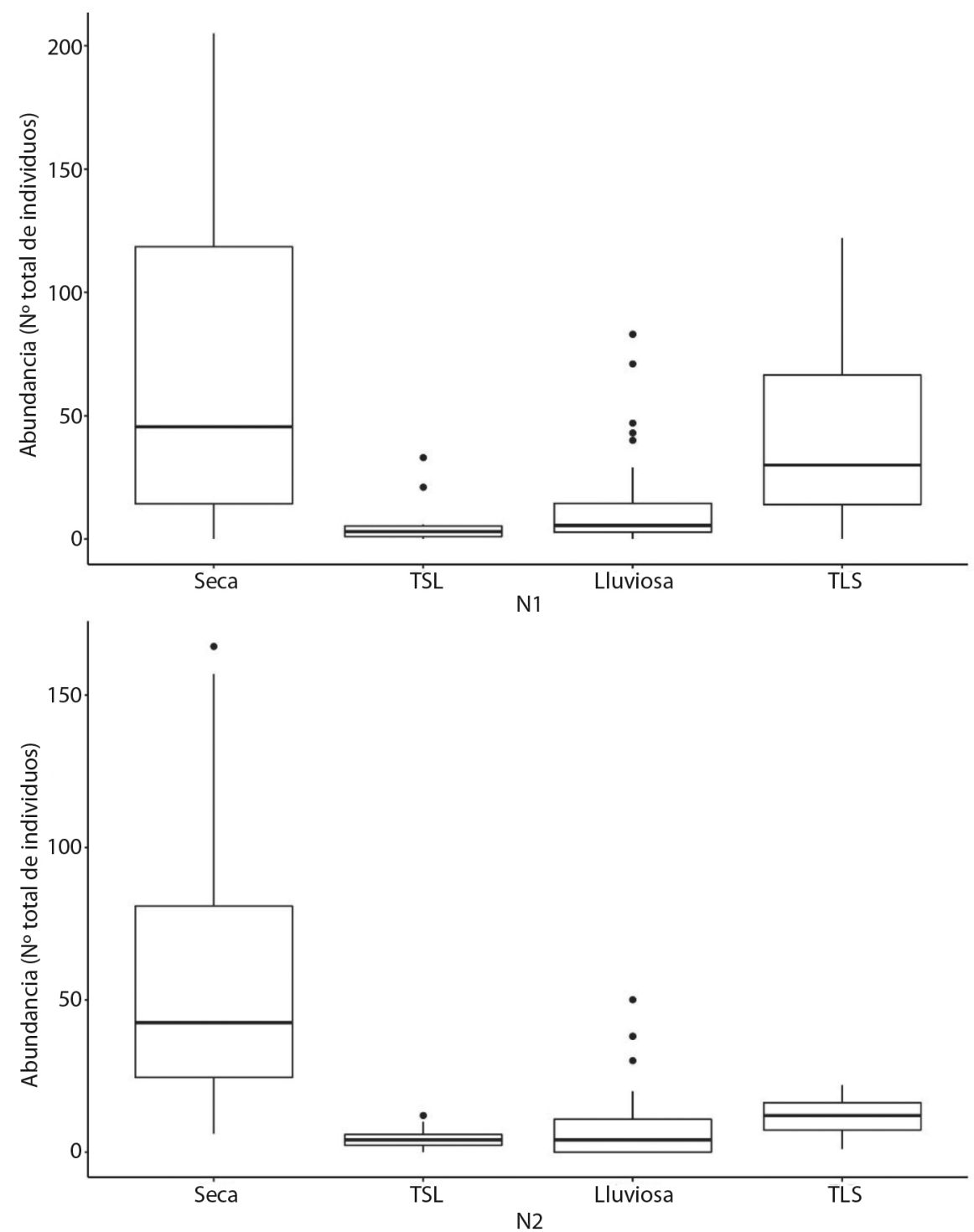

Fig. 3. Abundancia de macroinvertebrados acuáticos registrada en los cuatro periodos del año, en los dos sitios de muestreo en el río Naranjo, Costa Rica, 2017-2018, TSL: transición seca a lluviosa; TLS: transición lluviosa a seca.

Fig. 3. Aquatic macroinvertebrate abundance in the four periods of the year, in the two sampling sites in the Naranjo River, Costa Rica, 2017-2018, TSL: dry to wet transition; TLS: wet to dry transition.

diferencia significativa en la abundancia de macroinvertebrados acuáticos entre los sitios (PERMANOVA, $\mathrm{F}=2.1547 ; \mathrm{p}=0.07$ ) (Fig. 3).

El ACC indicó que la varianza entre las variables ambientales y biológicas puede ser explicada por los 2 primeros ejes en un $65 \%$. El primer eje presentó una relación positiva con el sustrato limo (0.45) y la arena (0.39) y negativa para las variables velocidad del agua (0.87) y el sustrato roca (-0.37). El segundo eje mostró una relación positiva con el caudal (0.79) y los nitratos $(0.58)$ y negativa para el sustrato canto rodado (-0.41). De esta forma y como ejemplo, algunos organismos tales como Paltostoma sp. 

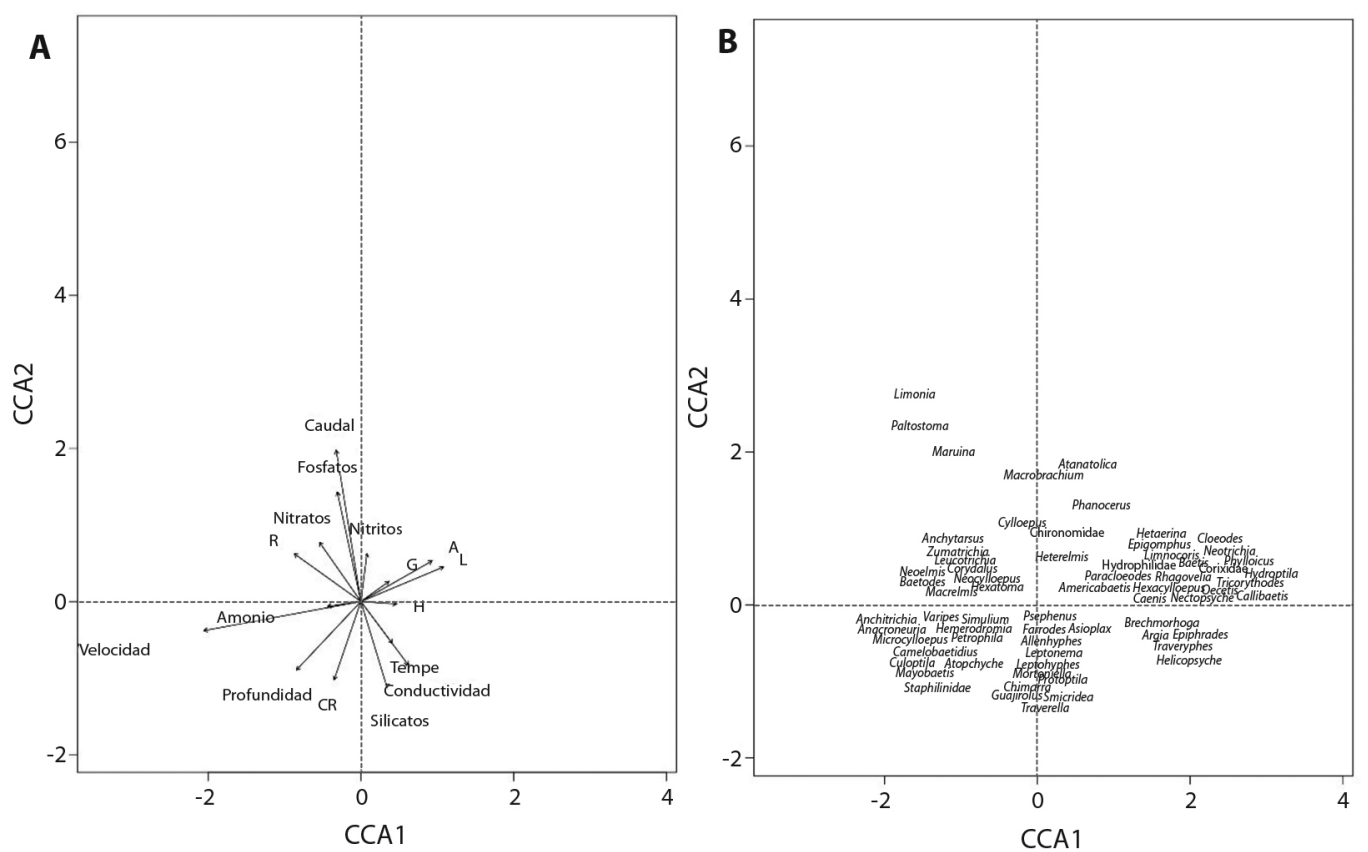

Fig. 4. Ordenación construida por el análisis de correspondencia canónica para los dos sitios de muestreo, A) ordenación de las variables físico, químicas e hidrológicas, B) ordenación de los géneros de macroinvertebrados acuáticos, Río Naranjo, Costa Rica, 2017-2018.

Fig. 4. Ordination plots constructed by canonical correspondence analysis for the two sampling places, A) Ordination of the physical, chemical and hydrological variables, B) ordination of the aquatic macroinvertebrates genera, Naranjo River, Costa Rica, 2017-2018.

(Diptera: Blephariceridae), Limonia sp. (Diptera: Tipulidae) y Maruina sp. (Diptera: Psychodidae), presentaron una relación con las variables caudal y los nitratos. Los géneros Callibaetis sp. y Paracloeodes sp. (Ephemeroptera: Baetidae), Epigomphus sp. (Odonata: Gomphidae) y Hetaerina sp. (Odonota:Calopterygidae) tienen afinidad por los sustratos grava, limo y arena. Se observó una relación con la velocidad del agua en los géneros Simulium sp. (Diptera: Simuliidae) y Anacroneuria sp. (Plecoptera: Perlidae) (Fig. 4).

La mayoría de los géneros presentaron una tendencia negativa ante un incremento del caudal y de los nitratos según la prueba de Spearman, únicamente los géneros Macrobrachium sp. (Decapoda: Palaemonidae), Paltostoma sp., Maruina sp. y la familia Chironomidae (Diptera) respondieron de manera positiva (aunque con una relación débil) al aumento del caudal.
Aumentos en la velocidad del agua y del sustrato roca generan una tendencia positiva (aunque débil) para el género Baetodes sp. (Ephemeroptera: Baetidae). Mientras que géneros como Callibaetis sp., Americabaetis sp. (Epehemeroptera: Baetidae), Nectopsyche sp. (Trichoptera: Leptoceridae) y la familia Hydrophilidae (Coleoptera), respondieron de manera positiva ante aumentos del sustrato limo (Fig. 5).

\section{DISCUSIÓN}

El presente estudio evidencia las variaciones en los ensamblajes de macroinvertebrados acuáticos durante los cuatro periodos del año, así como en las condiciones fisicoquímicas e hidrológicas en el Río Naranjo. Independientemente del período, el orden Ephemeroptera fue el más abundante. El cambio en el caudal del río pudo ser el principal factor 


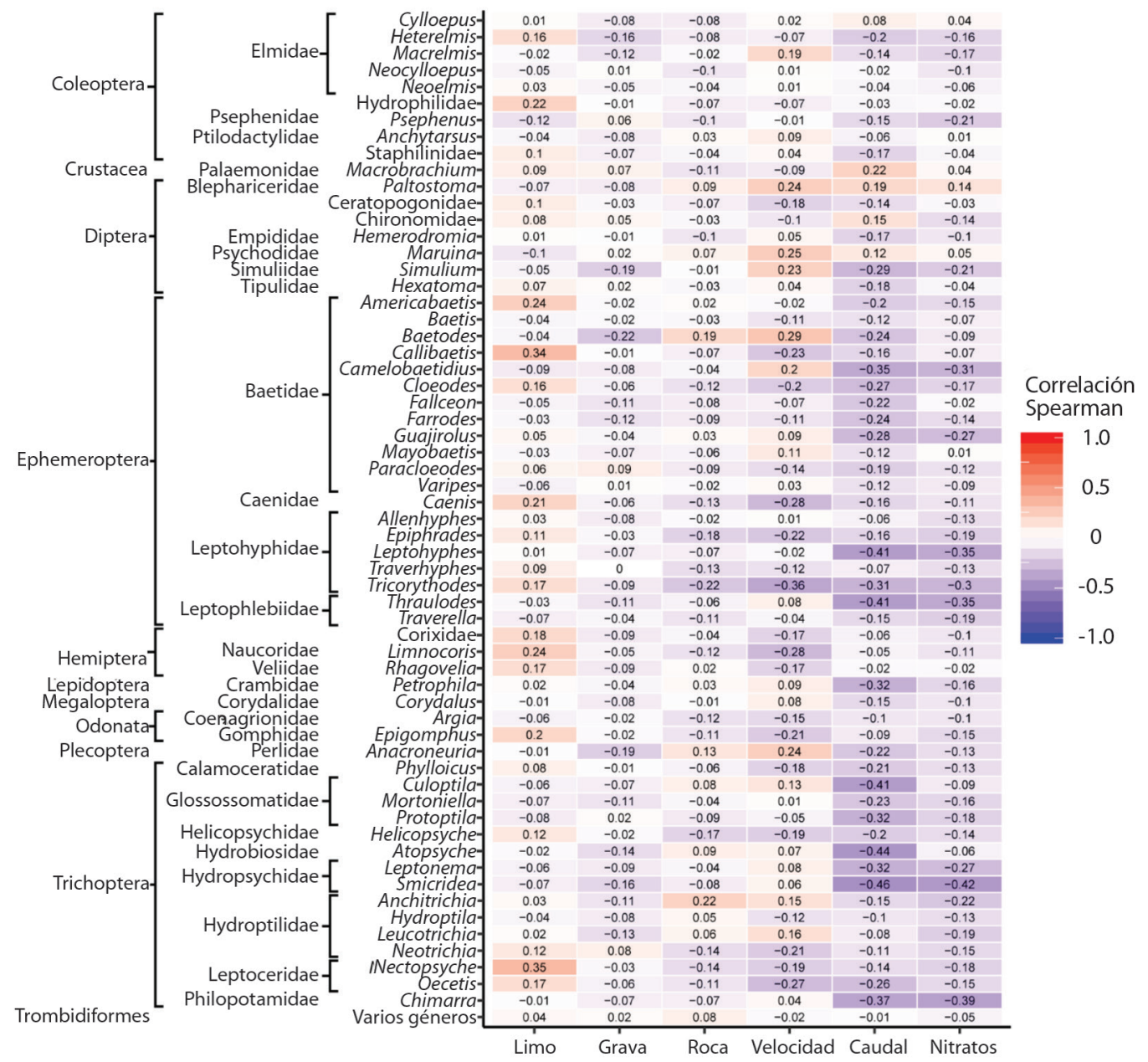

Fig. 5. Relación de los macroinvertebrados acuáticos con cinco variables en el Río Naranjo, Costa Rica, 2017-2018.

Fig. 5. Relationship of aquatic macroinvertebrates to five variables in the Naranjo River, Costa Rica, 2017-2018.

en la disminución en la abundancia de macroinvertebrados acuáticos debido a que la mayoría de los organismos respondieron de manera negativa ante el aumento del volumen de agua.

La alta abundancia del orden Ephemeroptera en ambos sitios puede deberse a que es uno de los órdenes acuáticos con mayor cantidad de familias y géneros, logrando colonizar los distintos hábitats que se originan en el río (Suhaila, Che \& Nurul, 2014). Además, la abundancia del género Camelobaetidius sp. puede deberse a que este es generalista en la selección del hábitat y ante cambios en las condiciones fisicoquímicas (Forero-Céspedes, Gutiérrez, \&
Reinoso-Flórez, 2016). En el caso del orden Trichoptera, su representatividad en el Río Naranjo, se debió a las larvas de los géneros Leptonema sp. y Smicridea sp. (Hydropsychidae), los cuales por su capacidad de construir refugios en sustratos como piedras, roca, grava y macrófitas les permite colonizar una mayor variedad de hábitats (Serna, Tamaris-Turizo \& Gutiérrez, 2015; Springer, 2010).

La abundancia en el orden Diptera en ambos sitios, se debió a la familia Chironomidae. Los representantes de esta familia pueden colonizar una gran variedad de macro y microhábitats, como, rápidos, pozas, madera 
sumergida, arena, grava, canto rodado y raíces; como también utilizar los sedimentos finos para generar refugios tubulares (Pinder, 1995; Oviedo-Machado \& Reinoso-Flórez, 2018).

El género Macrobrachium (Decapoda: Palaemonidae), fue registrado únicamente en el sitio correspondiente a cuenca media-baja, y por su comportamiento migratorio, se esperaba registrarlo en ambos sitios de muestreo, ya que según Bauer (2011), estos organismos pueden recorrer hasta varios kilómetros a lo largo de su etapa de vida. Su ausencia en el sitio N2 podría deberse a la temperatura del agua, ya que García, Becerril, Vega \& Espinosa (2013) sugieren que algunas especies de Macrobrachium son afectados en su tasa metabólica por las variaciones en la temperatura. Aun así, su ausencia en el sitio N2 es incierta y se deberían realizar estudios sobre la distribución altitudinal de estos crustáceos en la cuenca del Río Naranjo.

Por otra parte, las fluctuaciones en la abundancia de macroinvertebrados acuáticos entre los periodos del año son probablemente parte del dinamismo de la comunidad o bien consecuencia de los disturbios, por lo que se espera que existan variaciones temporales en la misma (McCord \& Kuhl, 2013). A pesar de los cambios que se dan entre los periodos del año, la comunidad puede ser resiliente en el sentido de que, la configuración original se restablece rápidamente después de las perturbaciones climatológicas e hidrológicas (Mesa, 2012).

La mayor abundancia durante el periodo seco se puede deber a que, ante la disminución de caudal se reduce la fuerza de arrastre, la corriente y el movimiento de sustratos de menor tamaño (Allan \& Castillo, 2007). Estas condiciones permiten el asentamiento de organismos que pueden sobrevivir a bajas velocidades y a la vez se mantiene el ensamblaje de organismos de hábitats rápidos y turbulentos (Worrall et al., 2014; Dunbar et al., 2010; Extence, Balbi \& Chadd, 1999), mientras se mantenga un caudal que permita la formación de dichos hábitats.

La relación contrapuesta entre los sustratos limo y arena con la velocidad del agua se debe a que, al disminuir la velocidad se disminuye el arrastre de sustratos de distintos tamaños. De esta forma en las pozas y en las orillas de los ríos, se acumulan los sustratos de menor tamaño como lo son la grava y el limo (Leopold, Wolman, \& Miller, 1992). Mientras que en las zonas con mayor turbulencia y fuerza de arrastre predominan los sustratos de mayor tamaño como el canto rodado y la roca (Leopold et al.,1992). De igual forma, debido a la velocidad del agua y al tipo de sustrato se van a registrar distintos macroinvertebrados acuáticos (Degani et al., 1993; Duan, Wang, \& Tian, 2008; Schoen, Merten, \& Wellnitz, 2013), en este caso relacionados al limo y a la arena, según el ACC (Fig. 4B), por ejemplo, se encuentran géneros como Limnocoris sp. (Hemiptera: Naucoridae), Epigomphus sp. (Odonata; Gomphidae), Nectopsyche sp. (Trichoptera: Leptoceridae), Caenis sp. (Ephemeroptera: Caenidae) y la familia Hydrophilidae (Coleoptera), todos estos frecuentemente reportados a bajas velocidades y en sustratos finos (Flowers \& de la Rosa, 2010; Oliva, 2014; Hanh, et al. 2018; Rodrigues \& Sites, 2019).

La relación -aunque débil- entre algunos géneros con el aumento de la velocidad se debe a sus adaptaciones fisiológicas y morfológicas. En el caso de organismos como Baetodes sp. (Ephemeroptera: Baetidae) y Anacroneuria sp. (Plecoptera: Perlidae) cuentan con uñas fuertes en forma de gancho en cada par de patas y cuerpos aplanados (Flowers \& de la Rosa, 2010; Gutiérrez-Fonseca, 2010), mientras que Paltostoma sp. (Diptera: Blephariceridae), Simulium sp. (Diptera: Simuliidae) y Maruina sp. (Diptera: Psychodidae) poseen ventosas para aferrarse al sustrato y evitar ser arrastrados por la corriente (Gil \& Da Santos, 2016; Hamada, Oliveira, Pepinelli, \& Ribeiro, 2014). De igual forma, estos mismos organismos presentaron una respuesta positiva para el sustrato tipo roca (Fig. 5), ya que existe una relación entre la velocidad del agua y el tipo de sustrato (Allan $\&$ Castillo, 2007). Con forme aumenta el tamaño del sustrato, aumenta la turbulencia y se reduce la profundidad del agua (Hill, Maddock, \& Bickerton, 2013). Mientras que, y como se mencionó anteriormente, en sustratos como 
la grava, la velocidad suele ser menor y por esta razón los organismos considerados reófilos, presentaron valores negativos para este tipo de sustrato con la prueba de Spearman.

La mayoría de los macroinvertebrados acuáticos respondieron de manera negativa al aumento del caudal, esto debido a que conforme aumenta el volumen de agua, aumenta la profundidad y la fuerza de arrastre (Gordon, McMahon, \& Finlayson 2004) y no todos los organismos registrados en el Río Naranjo cuentan con características para sobrevivir ante estas condiciones. De esta forma, durante el periodo lluvioso y con mayor aumento de caudal, se registraron principalmente a los géneros Maruina sp., Paltosotoma sp., Anacroneuria sp. y Baetodes sp., por sus adaptaciones anteriormente citadas; también los géneros Anchitrichia sp. (Trichoptera: Hydroptilidae) y Petrophila sp. (Lepidoptera: Crambidae) ya que construyen refugios de seda para evitar ser arrastrados por la corriente (Springer, 2010; Tuskes \& Mcgowan-Tuskes, 2019).

Por último, durante el periodo lluvioso es normal que las condiciones fisicoquímicas e hidráulicas fluctúen y estas pueden variar de mayor a menor escala dependiendo del tipo de actividades humanas que se encuentren en su alrededor (Gao \& Ruan, 2017). Harding, Young, Hayes, Shearer \& Stark (1999), mencionan que, un aumento en la concentración de nitratos puede causar una disminución de los organismos más sensibles como Ephemeroptera, Plecoptera y Trichoptera (EPT). Sin embargo, en el Río Naranjo, se mantuvo la presencia de estos ordenes durante todo el año de muestreo, dando a entender que la variación de nitratos no fue lo suficientemente grave como para afectarlos o que las especies presentes tienen un grado de tolerancia flexible a esta variable.

Las variaciones fisicoquímicas y de caudal registradas en el Río Naranjo, permiten variar el ensamblaje de macroinvertebrados acuáticos, entre periodos, por lo que, se observa es una condición típica de ríos tropicales con una disminución en la abundancia durante los meses con mayores precipitaciones (Zamora, 2015). Así, se puede concluir que el caudal del río puede ser el principal factor que genera la variación en la abundancia de macroinvertebrados acuáticos en el Río Naranjo. A la vez se resalta que estas variaciones son naturales y por ende se deben mantener, junto con la preservación del bosque de ribera a lo largo del río, para evitar que a futuro las fluctuaciones en los ensamblajes de macroinvertebrados acuáticos se den por condiciones antrópicas.

Declaración de ética: los autores declaran que están de acuerdo con esta publicación; que no existe conflicto de interés de ningún tipo; y que ha cumplido con todos los requisitos y procedimientos éticos y legales pertinentes. Todas las fuentes de financiamiento se detallan plena y claramente en la sección de agradecimientos. El respectivo documento legal firmado se encuentra en los archivos de la revista.

\section{AGRADECIMIENTOS}

Se agradece al Instituto Costarricense de Electricidad (ICE), por los datos de caudal del Río Naranjo. A Juan Guillermo Sagot (CIMAR) por los datos fisicoquímicos y a Randall Jiménez por la colaboración en la estadística.

\section{RESUMEN}

Introducción: La distribución de los macroinvertebrados acuáticos en los ríos puede estar determinada por el tipo del sustrato, la forma del canal del río, el régimen hidrológico y las interacciones con factores bióticos y abióticos. En Costa Rica se desconoce en gran parte de los ríos, las variaciones en la distribución de los macroinvertebrados acuáticos producto de las transiciones y consecuentes variaciones en características fisicoquímicas e hidrológicas. Objetivos: 1) Estudiar el cambio del ensamblaje de macroinvertebrados acuáticos en dos sitios dentro del cauce del Río Naranjo, durante los cuatro periodos hidrológicos del año; 2) determinar la relación entre las variables fisicoquímicas, sustrato y el caudal con el ensamblaje. Metodología: El estudio se realizó en el Río Naranjo, Costa Rica, en dos sitios de muestreo durante un año, evaluando los cuatro periodos hidrológicos del año. En cada sitio de muestreo se obtuvieron valores de velocidad y profundidad del agua, caudal, oxígeno disuelto, $\mathrm{pH}$, conductividad, sulfatos, amonio, nitrato, silicatos, fosfatos y sustrato, y se obtuvo una muestra de macroinvertebrados acuáticos por cada tipo de microhábitat determinado 
por la velocidad del agua. Se realizó una prueba PERMANOVA de dos vías, para determinar si existe una diferencia entre la abundancia de macroinvertebrados acuáticos, por periodo y entre sitios. Se hizo un análisis de correspondencia canónica para observar la relación entre los macroinvertebrados acuáticos y los factores ambientales. Por último, se realizó una prueba de Spearman para determinar la relación positiva o negativa de las variables con los macroinvertebrados Resultados: Hubo una mayor abundancia durante la estación seca, mientras que durante la transición de la estación seca a la lluviosa se registró la menor abundancia de organismos. El ACC indicó que el primer eje presentó una relación positiva con el sustrato limo y arena, y una relación negativa para las variables velocidad de corriente y sustrato rocoso. El segundo eje presentó una relación positiva con el caudal y los nitratos, y negativa para el sustrato canto rodado. La mayoría de los organismos mostraron una respuesta negativa al aumento del flujo y los nitratos. Discusión: Conforme aumenta el caudal, aumenta la velocidad del agua y no todos los organismos registrados en el Río Naranjo cuentan con características para sobrevivir a estas condiciones. Además, la profundidad también aumenta junto con la fuerza de arrastre, provocando el movimiento de los sustratos de menor tamaño y una remoción de organismos. El aumento de nitratos puede generar una disminución de los organismos más sensibles como Plecoptera y Ephemeroptera. A pesar de las variaciones físicoquímicas y de caudal registradas en el Río Naranjo, éstas no fueron lo suficientemente fuertes como para variar el ensamblaje de macroinvertebrados acuáticos, por lo que la cuenca por el momento no cuenta con factores estresantes que elimine organismos durante uno o varios meses del año.

Palabras clave: caudal; velocidad del agua; sustrato; insectos acuáticos; ensamblaje.

\section{REFERENCIAS}

Allan, D., \& Castillo, M. (2007). Stream ecology: Structure and function of running waters. 2nd Edition, Chapman and Hall, New York.

Appalasamy, S., Arumugam, N., Sukri, S., \& Rak, A. E. (2018). Physico-chemical water quality and macroinvertebrate distribution along Sungai Asah in Pulau Tioman, Johor, Malaysia. Songklanakarin Journal of Science and Technology, 40(6), 1265-1270.

Bauer, R. (2011). Amphidromy and migrations of freshwater shrimps. II. Delivery of hatching larvae to the sea, return of juvenile upstream migration and human impacts. In: A. Asakura (Ed.). New frontiers in crustacean biology. Proceedings of the Crustacean Society Summer Meeting, Tokyo, Brill, Leiden.

Cauvy-Fraunié, S., Andino, P., Espinosa, R., Calvez, R., Jacobsen, D., \& Dangles, O. (2016). Ecological responses to experimental glacier-runoff reduction in alpine rivers. Nature Communications, 7, 1-7.

Contreras-Ramos, A., \& Harris, S. (1998). The Immature Stages of Platyneuromus (Corydalidae), with a Key to the Genera of Larval Megaloptera of Mexico. Journal of the North American Benthological Society, 17(4), 489-517.

Degani, G., Herbst, G., Rotal, R., Bromley, H., Levanon, D., Netzer, Y., Harari, N., \& Glazman, H. (1993). Relationship between current velocity depth and the invertebrate community in a stable river system. Hydrobiologia, 263(1), 163-172

Duan, X., Wang, Z., \& Tian, S. (2008). Effect of streambed substrate on macroinvertebrate biodiversity. Frontiers of Environmental Science \& Engineering in China, 2(1),122-128.

Dunbar, M. J., Pedersen, M. L., Cadman, D., Extence, C., Waddingham, J., Chadd, R., \& Larsen, S. E. (2010). River discharge and local-scale physical habitat influence macroinvertebrate LIFE scores. Freshwater Biology, 55(1), 226-242.

Extence, C. A., Balbi, D. M., \& Chadd, R. P. (1999). River Flow Indexing using British Benthic Macroinvertebrates: a framework for setting hydroecological objectives. Regulated Rivers Research \& Management, 15(6), 543-574.

Ferru, M., \& Fierro, P. (2015). Estructura de macroinvertebrados acuáticos y grupos funcionales tróficos en la cuenca del río Lluta, desierto de Atacama, Arica Y Parinacota, Chile. Idesia, 33(4), 47-54.

Flowers, W., \& De la Rosa, C. (2010). Ephemeroptera. En: Springer, M., Ramírez, A. \& Hanson, P. (Eds). Macroinvertebrados de agua dulce de Costa Rica I. Revista de Biología Tropical, 58(4), 63-93.

Fonnesu, A., Sabetta, L., \& Basset, A. (2005). Factors affecting macroinvertebrate distribution in a mediterranean intermittent stream. Journal of Freshwater Ecology, 20 (4), 641-647.

Forero-Céspedes, A., Gutiérrez, C., \& Reinoso-Flórez, G. (2016). Composición y estructura de la familia Baetidae (Insecta: Ephemeroptera) en una cuenca andina colombiana. Hidrobiológica, 26(3), 459-474.

Gao, C., \& Ruan, T. (2017). The influence of climate change and human activities on runoff in the middle reaches of the Huaihe River Basin, China. Journal of Geographical Sciences, 28(1), 79-92.

García-Guerrero, M., Becerril-Morales, F., Vega-Villasante, F., \& Esponosa-Chaurand, L. (2013). Los langostinos del género Macrobrachium con importancia económica y pesquera en América Latina: conocimiento actual, rol ecológico y conservación. Latin American Journal of Aquatic Research, 41(4), 651-675. 
Gil-Azevedo, L. \& Da Santos, D. (2016). Family Blephariceridae. Zootaxa, 4122(1), 182-186.

Gordon, N., McMahon, T., \& Finlayson, B. (2004). Stream hydrology an introduction for ecologists. John Wiley \& Sons Ltd, West Sussex, England.

Hamada, N., Oliveria, J., Pepinelli, M., \& Ribeiro, L. (2014). Ordem Diptera. En: Hamada, N., Nessimian, J., \& Barbosa, R. (Eds). Insetos Aquáticos na Amazonia brasileira: taxonomia, biologia, e ecologia. Instituto Nacional de Pesquisas da Amazonia, Brasil.

Hanh, T., Eurie, M., Boets, P., Lock, K., Damanik, M., Suhareva, N., Everaert, G., Van der heyden, C., Domínguez-Granada, L., Thi, T., \& Goethals, P. (2018). Threshold responses of macroinvertebrate communities to stream velocity in relation to hydropower dam: A case study from the Guayas River basin (Ecuador). Water, 10(1), 1-17.

Harding, JS., Young, RG., Hayes, JW, Shearer, KA., \& Stark, JD. (1999). Changes in agriculture intensity and river health along river continuum. Freshwater Biology, 42, 345-357.

Harinkhede, P., \& Manekar, A. (2016). Correlation aspects between macroinvertebrates and physicochemical factors to assess water quality of the River Vainganga. International Journal of Science and Research, 5 (8), 1582-1585.

Hill, G., Maddock, I., \& Bickerton, M. (2013). Testing the relationship between surface flow types and benthic macroinvertebrates. In: Maddock, I, Harby, A., Kemp, P., \& Wood, P. (Eds). Ecohydraulics. Wiley Blackwell. UK.

Horrigan, N., \& Baird, D.J., (2008). Trait patterns of aquatic insects across gradients of flowrelated factors: a multivariate analysis of Canadian national data. Canadian Journal of Fisheries and Aquatic Sciences, 65, 670-680.

Kail, J., Arle, J., \& Jähnig, S. C. (2012). Limiting factors and thresholds for macroinvertebrate assemblages in European rivers: Empirical evidence from three datasets on water quality, catchment urbanization, and river restoration. Ecological Indicators, 18(1), 63-72.

Kassambara, A. (2019). Visualization of a Correlation Matrix using 'ggplot2'. R package version 0.1.3.

Kubendran, T., \& Ramesh, M. (2016). Composition and distribution of aquatic insect communities in relation to water quality in two freshwater streams of southern western ghats, India. Journal of Entomology and Zoology Studies, 4(5), 689-695.

Leopold, L., Wolman, G., \& Miller, J. 1992. Fluvial processes in geomorphology. Dover Publication, New York, USA.
Manzo, V., \& Archangelsky, M. (2008). A key to the known larvae of South American Elmidae (Coleoptera: Byrrhoidea), with a description of the mature larva of Macrelmis saltensis, Manzo. Annales de Limnologie - International Journal of Limnology, 44(1), 63-74.

McCord, S., \& Kuhl, B. (2013). Macroinvertebrate community structure and its seasonal variation in the Upper Mississippi River, USA: a case study. Journal of Freshwater Ecology, 28(1), 63-78.

Mengzhen, X., Zhaoyin, W., Xuehua, D., \& Baozhu, P. (2014). Effects of pollution on macroinvertebrates and water quality bio-assessment. Hydrobiologia, 729(1), 247-259.

Mesa, L. (2012). Interannual and seasonal variability of macroinvertebrates in monsoonal climate streams. Brazilian Archives of Biology and Technology, 55(3), 403-410.

Molina, C., Gibon, F., Pinto, J., \& Rosales, C. (2008). Estructura de macroinvertebrados acuáticos en un río altoandino de la cordillera real, Bolivia: variación anual y longitudinal en relación a factores ambientales. Ecología Aplicada, 7 (1,2): 1-12.

Oksanen, J., Guillaume, F., Friendly, M., Kindt, R., Legendre, P., McGlinn, D., Minchin, P., O’Hara, R., Simpson, G., Solymos, P., Stevens H., Szoecs E., \& Wagner, H. (2019). Vegan: Community Ecology Package. R package version 2.5-5.

Oliva, A. (2014). Distribution of the species of Berosini (Coleoptera: Hydrophilidae) in South America. Revista Colombiana de Entomología, 40(2), 272-280.

Oviedo-Macho, N., \& Reinoso-Flórez, R. (2018). Aspectos ecológicos de larvas de Chironomidae (Diptera), del río Opia (Tolima, Colombia). Revista Colombiana de Entomología, 44(1), 101-109.

Pinder, L., (1995). The habitats of chironomid larvae. In P., Armitage, P., Cranston, \& L. Pinder (Eds), The Chironomidae (pp. 107-135). Springer, Dordrecht.

R Core Team (2019). R: A language and environment for statistical computing. R Foundation for Statistical Computing, Vienna, Austria. https:// www.R-project.org/

Ramírez, A. (2010). Odonata. En: Springer, M., Ramírez, A. \& Hanson, P. (Eds). Macroinvertebrados de agua dulce de Costa Rica I. Revista de Biología Tropical, 58(4):97-136.

Rodrigues, H., \& Sites, R. (2019). Revision of Limnocoris (Heteroptera: Nepomorpha: Naucoridae) of North America. Zootaxa, 4629(4), 451-497.

Roldán, G. (1998). Guía para el estudio de los macroinvertebrados acuáticos del Departamento de Antioquia, Pama Editores Ltda. Bogotá, Colombia. 
Roldán-Pérez, G. (2016). Los macroinvertebrados acuáticos como indicadores de la calidad del agua: cuatro décadas de desarrollo en Colombia y latinoamérica. Revista de la Academia Colombiana de Ciencias Exactas, Físicas y Naturales, 40(155): 254-274.

Schoen, J., Merten, E., \& Wellnitz, T. (2013). Current velocity as a factor in determining macroinvertebrate assemblages on wood surfaces. Journal of Freshwater Ecology, 28(2), 271-275.

Sefick, S. A., Kosnicki, E., Paller, M. H., \& Feminella, J. W. (2018). Hydrogeomorphic reference conditions and its relationship with macroinvertebrate assemblages in Southeastern US Sand Hills streams. Journal of the American Water Resources Association, 54(9), 1-18

Serna, D., Tamaris-Turizo, C., \& Gutiérrez, L. (2015). Distribución espacial y temporal de las larvas de Trichoptera (Insecta) en el río Manzanares, Sierra Nevada de Santa Marta (Colombia). Revista Biología Tropical, 63(2), 465-477.

Springer, M. (2010). Trichoptera. En: Springer, M., Ramírez, A. \& Hanson, P. (Eds). Macroinvertebrados de agua dulce de Costa Rica I. Revista de Biología Tropical, 58(4), 63-93.

Springer, M. (2019). La colección de Entomología Acuática del Museo de Zoología, Universidad de Costa Rica: lista actualizada de géneros, importancia y retos futuros. Revista Biología Tropical, 67(2), 200-211.
Suhaila, A., Che, M., \& Nurul, H. (2014). Seasonal abundance and diversity of aquatic insects in rivers in Gunung Jerai Forest Reserve, Malaysia. Sains Malaysiana, 43(5), 667-674.

Syvitski, J., Cohenb, S., Kettner, A., \& Brakenridge, G. (2014). How important and different are tropical rivers? - An overview. Geomorphology, 227(1), 5-17

Tuskes, P., \& Mcgowan-Tuskes, A. (2019). Aquatic Moths of the Genus Petrophila and their Biology in Oak Creek, Arizona (Crambidae). The Journal of the Lepidopterists Society, 73(1), 43-53.

Vörösmarty, C.J., McIntyre, P.B., Gessner, M. O., Dudgeon, D., Prusevich, A., Green, P., Glidden, S., Bunn, S.E., Sullivan, C.A., Reidy, C., \& Davies, P. (2010). Global threats to human water security and river biodiversity. Nature, 467 (1), 555-561.

Wickham, H. (2016). ggplot2: Elegant Graphics for Data Analysis. Springer-Verlag New York.

Worrall, T. P., Dunbar, M. J., Extence, C. A., Laizé, C. L. R., Monk, W. A., \& Wood, P. J. (2014). The identification of hydrological indices for the characterization of macroinvertebrate community response to flow regime variability of macroinvertebrate community response to flow regime variability. Hydrological Sciences Journal, 59(3-4), 645-658.

Zamora, H. (2015). Macroinvertebrados acuáticos registrados durante la época de lluvias en tres ríos del piedemonte llanero colombiano. Revista Colombiana de Ciencia Animal, 7(2), 139-147. 